\title{
Spectral and mineralogical characterization of inner main-belt V-type asteroids ${ }^{\star}$
}

\author{
M. C. De Sanctis ${ }^{1}$, A. Migliorini ${ }^{1}$, F. Luzia Jasmin², D. Lazzaro ${ }^{2}$, G. Filacchione ${ }^{1}$, S. Marchi ${ }^{3}$, \\ E. Ammannito ${ }^{4}$, and M. T. Capria ${ }^{1}$ \\ ${ }^{1}$ Istituto di Astrofisica Spaziale e Fisica Cosmica, INAF, Area di Ricerca Tor Vergata, via del Fosso del Cavaliere, 100, \\ 00133 Roma, Italy \\ e-mail: mariacristina.desanctis@iasf-roma.inaf.it \\ 2 Observatório Nacional, Rua Gal José Cristino 77, 20921-400 Rio de Janeiro, Brazil \\ 3 Département Cassiopée, Université de Nice-Sophia Antipolis, Observatoire de la Côte d'Azur, CNRS, Boulevard de l'Observatoire, \\ 06304 Nice, France \\ ${ }^{4}$ Istituto di Fisica dello Spazio Interplanetario, Area di Ricerca Tor Vergata, via del Fosso del Cavaliere, 100, 00133 Roma, Italy
}

Received 26 April 2011 / Accepted 14 July 2011

\begin{abstract}
Context. V-type asteroids in the inner main belt are thought to be genetically related to (4) Vesta as collisional fragments. We investigate their relationship with Vesta observing putative V-type asteroids.

Aims. The aim of this work is to observe candidate V-type asteroids, selected in different regions of the inner main belt, to characterize them and hence better understand their relationship with (4) Vesta.

Methods. We present new NIR reflectance spectra of 18 V-type candidate asteroids, selected from datasets of possible V-type asteroids. The data were obtained at the $3.6 \mathrm{~m}$ Telescopio Nazionale Galileo, covering the spectral range 0.7 to $2.5 \mu \mathrm{m}$. We derive spectral parameters from NIR spectra to infer mineralogical information of the observed asteroids. The spectra of these asteroids are examined and compared to those of Howardite-Eucrite-Diogenite meteorites (HED), of which (4) Vesta is believed to be the parent body, and other V-type asteroids observed during previous campaigns. To enlarge the data set and increase the statistical significance of the analysis, we included the data presented in our previous article, obtaining a final data set of $41 \mathrm{~V}$-type asteroids.

Results. The V-type asteroids examined here show a higher variability of band parameters with respect to HEDs values, as for (4) Vesta. This result indicates that (4) Vesta and V-type asteroids have mineralogies that are not completely represented in the HEDs collection.

Conclusions. An important finding is that some of the observed asteroids show spectral characteristics similar to diogenites, a result that may have relevant implications on their origin. Moreover, most of the diogenitic asteroids are not members of the Vesta classical family.
\end{abstract}

Key words. minor planets, asteroids: general - minor planets, asteroids: individual: Vesta - techniques: spectroscopic

\section{Introduction}

Basaltic asteroids are believed to derive from bodies whose interiors reached the melting temperature of silicate rocks and subsequently differentiated (Gaffey et al. 2002). For a long time (4) Vesta was the only known asteroid presenting a basaltic crust (McCord et al. 1970; McFadden et al. 1977; Larson \& Fink 1975; Gaffey 1997; Binzel et al. 1997) but in recent years an increasingly large number of small asteroids with a similar surface composition have been discovered. The discovery of new objects far away from Vesta but taxonomically classified as V-type asteroids, poses the problem regarding the provenance of basaltic material in the asteroid main belt. Although some of them can be directly linked to (4) Vesta, forming its dynamical family, others do not appear to have a clear dynamical link, suggesting, thus, the existence of other basaltic parent bodies. In particular, the discovery of small basaltic asteroids in the outer and

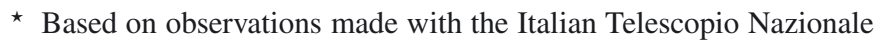
Galileo (TNG) operated on the island of La Palma by the Centro Galileo Galilei of the INAF (Istituto Nazionale di Astrofisica) at the Spanish Observatorio del Roque de los Muchachos of the Instituto de Astrofisica de Canarias. intermediate main belt (Lazzaro et al. 2000; Binzel et al. 2006; Hammergren et al. 2006; Moskovitz et al. 2008; Roig et al. 2008; Duffard \& Roig 2009), as well as in the inner belt, but far away from the dynamical limits of the Vesta family (e.g. Xu et al. 1995; Duffard et al. 2004; Alvarez-Candal et al. 2006), confirms that several differentiated parent bodies must have formed and proves that some of their basaltic material has survived over the life of the solar system.

A strong indication of the presence of multiple large basaltic asteroids, at least in the early phases of our solar system, arises also from diverse studies of the Howardite-Eucrite-Diogenite meteorites (HED). The HEDs are basaltic meteorites and are thought to come from large asteroids that melted to form a metallic core and basaltic magmas after the formation. A close connection between asteroid (4) Vesta and these basaltic achondrites was established during the earliest studies, including visible and infrared spectroscopic investigations (McCoord et al. 1970; McFadden et al. 1977; Xu et al. 1995; Burbine et al. 2001), numerical and dynamical simulations (Asphaug 1997), and spatial resolved observations (Binzel et al. 1997; Thomas et al. 1997). However, recent studies in oxygen isotopic composition from a common mass-fractionation line suggest that a few eucrite-like 
meteorites come from other bodies, or that (4) Vesta was not completely homogenized during differentiation (McSween et al. 2010). It is noteworthy that a laboratory analysis of iron meteorites also indicates an origin from at least 70 distinct differentiated parent bodies (Mittlefehldt et al. 1998). In addition, searches for meteorites in Antarctica resulting in new types of differentiated meteorites such as GRA 06128 and 06129 (Arai et al. 2008) suggest that multiple differentiated planetesimals existed in the early solar system. These studies indicate that basaltic material was common in the inner solar system, but it is not clear if there are currently any basaltic remnants of the putative differentiated parent bodies that once existed.

Several methods have been developed and applied to photometric surveys, in particular the SDSS-Moving Object Catalog MOC (Ivezic et al. 2001), to select candidate basaltic asteroids (Roig \& Gil-Hutton 2006; Moskovitz et al. 2008; Carvano et al. 2010). The distribution of candidate basaltic asteroids indicates that (4) Vesta is the predominant contributor to the basaltic material in the main belt because they cluster around the Vesta family. However, V-types, confirmed and putative, are widely distributed across the entire main belt, which is a strong indication that not all of them are to be linked to (4) Vesta (e.g., Florczak et al. 2002; Duffard et al. 2004; Alvarez-Candal et al. 2006).

The origin of these objects is debated. According to hydrocode simulation, Asphaug (1997) found that multikilometer fragments can be ejected from (4) Vesta with speeds up to $\Delta V=$ $550 \mathrm{~m} / \mathrm{s}$. From Gauss equations these $\Delta V$ values would lead to orbital changes up to $\approx 0.15 \mathrm{AU}, \approx 0.03$ and $\approx 1^{\circ}$ in semi-major axis, eccentricity, $e$, and inclination, $i$, respectively (Nesvorný et al. 2008). However, many candidate V-ype asteroids are displaced from (4) Vesta by up to 0.09 and $3^{\circ}$ in proper $e$ and $i$, respectively. On the other hand, it is important to mention that the dynamical boundaries of collisional families are not very clearly defined so that it is possible to find the same objects classified in or outside the Vesta family according to the selection criteria.

Some of the asteroids outside the Vesta family could be former family members that migrated to their current positions via Yarkovsky effect and nonlinear secular and mean-motion resonances (Carruba et al. 2005). Dynamical simulations by Nesvorný et al. (2008) show that most basaltic asteroids with $a<2.3$ AU located outside the Vesta family's borders are likely fugitives from the Vesta family that have evolved to their current orbits via various identified dynamical pathways. However, these simulations do not predict the orbits of many basaltic asteroids with $2.3<a<2.5 \mathrm{AU}$ and low inclinations, $i<6^{\circ}$.

Nesvorný et al. (2008) suggest that either these asteroids originated from (4) Vesta before the late heavy bombardment or they are fragments from other differentiated bodies. The latter alternative would set an important constraint on our understanding of differentiation processes in the early solar system.

Several basaltic asteroids have been discovered with $a>2.5 \mathrm{AU}$, among which we can mention (1459) Magnya (Lazzaro et al. 2000), (21238) 1995 WV7 (Hammergren et al. 2006; Binzel et al. 2006), (40521) 1999 RL95 (Roig et al. 2008), (10537) 1991 RY16 and (7472) Kumakiri (Duffard \& Roig 2009). All of these seem to have no connection to (4) Vesta not only because of their dynamical characteristics but also because of their spectra and derived mineralogies (Hardersen et al. 2004; Moskovitz et al. 2008; De Sanctis et al. 2011; Burbine et al. 2011). Therefore, it is important to understand if other V-type candidates also show distinct mineralogy with respect to (4) Vesta and its family members.

Previous works (Florczak et al. 2002) suggested that the spectra of V-type asteroids not members of the Vesta dynamical family have the $1 \mu \mathrm{m}$ band deeper than the family members and (4) Vesta itself. This characteristic would imply that two distinct mineralogical groups, either probing different layers of (4) Vesta or coming from different bodies, would coexist, as already suggested by Vilas et al. (2000).

Duffard et al. (2004) analyzed the properties of basaltic asteroids belonging to the Vesta family and in its neighborhood, showing again the possible presence of distinct mineralogies. However, their results were not conclusive. The recent work by Moskovitz et al. (2010), on the other hand, found that amongst the inner main-belt asteroids there is no evidence for non-Vestoid mineralogies. Instead, according to their analysis, these asteroids seem to represent a continuum of compositions, consistent with a common origin from a single differentiated parent body. De Sanctis et al. (2011) also found no clear differences between (4) Vesta, Vesta family and non-family objects. However, they reported that most of the observed V-ype asteroids show a low abundance of $\mathrm{Ca}(<10 \%$ Wo) with a predominance of diogenitic mineralogies among the non-family members. The spectral diversity between V-type asteroids belonging to the Vesta family and unrelated to it, has been recently addressed by Marchi et al. (2010) who found differences in the spectral slopes trends possibly caused by the presence of diogenite-rich asteroids.

To better constrain the problem of the origin of basaltic material in the main belt, in this work we will examine NIR spectra of candidate basaltic asteroids located in different regions of the main belt to see if there are spectral characteristics that enable us to distinguish between Vesta and non-Vesta related objects, which would then be indicative of different origins.

\section{Observations and data reduction}

We selected 18 asteroids among those classified as putative V-type, based on their SDSS colors (Iczevic et al. 2001), by Roig et al. (2006) and Carvano et al. (2010). Most of them are nonfamily members, according to the Nesvorný et al. (2008) definition. The mean orbital parameters, taken from the astdys web site (http://hamilton.dm.unipi.it/astdys/), the absolute magnitude and the diameter, obtained assuming an albedo of 0.3 as in Marchi et al. (2010), are provided in Table 1.

Near-infrared spectra of the selected asteroids have been acquired with the Telescopio Nazionale Galileo (TNG), a $3.6 \mathrm{~m}$ class telescope in La Palma, during a six-nights program on March 16-21 2010, and on a dedicated campaign using the Discretional Director Time (DDT) on November 2009 (Table 2). We used the Near-Infrared Camera Spectrometer (NICS) equipped with the Amici grism, and a 2-arcsec slit. The NICS offers a unique, high throughput, low-resolution spectroscopic mode with an Amici grism disperser, which yields a complete $0.8-2.5 \mu \mathrm{m}$ spectrum in one single acquisition. The low resolution together with the high efficiency of the Amici grism ( $90 \%$ across the entire infrared range) allowed us to obtain spectra of faint objects like small V-type asteroids with the advantage of having the whole near infrared range measured simultaneously. Three standard solar analog stars (Land 98-978, Land 102-1081, Land 107-998) were also observed during the night.

Data reduction was carried out according to the reduction techniques described in Licandro et al. (2002), Duffard et al. (2004), and Lazzarin et al. (2004), for example. We refer the reader to these articles for details. The asteroid spectra were finally normalized to achieve a reflectance value equal to 1 around $1.6 \mu \mathrm{m}$. 
M. C. De Sanctis et al.: Mineralogical characterization of V-type asteroids

Table 1. Orbital parameters, absolute magnitude, diameter and dynamical classification for each observed asteroid.

\begin{tabular}{lcccccc}
\hline \hline Asteroid & $a(\mathrm{AU})$ & $e$ & $i\left(^{\circ}\right)$ & $\mathrm{H}(\mathrm{mag})$ & Diam. $(\mathrm{km})$ & Vesta family \\
\hline 4383 Suruga & 2.42 & 0.055 & 6.275 & 12.8 & 6.7 & No \\
5498 Gustafsson & 2.24 & 0.098 & 2.786 & 13.9 & 4.0 & No \\
5560 Amytis & 2.28 & 0.118 & 4.824 & 13.5 & 4.8 & No \\
6563 Steinheim & 2.29 & 0.097 & 5.745 & 14.0 & 3.8 & No \\
6976 Kanatsu & 2.33 & 0.120 & 7.475 & 12.9 & 6.4 & No \\
8761 Crane & 2.24 & 0.096 & 3.675 & 13.6 & 4.6 & No \\
9147 Kourakuen & 2.19 & 0.108 & 6.892 & 13.4 & 5.1 & No \\
9531 Jean Luc & 2.23 & 0.138 & 5.492 & 13.7 & 4.4 & No \\
10614 1997UH1 & 2.35 & 0.089 & 6.482 & 13.6 & 4.6 & Yes \\
11764 Benbaillaud & 2.19 & 0.070 & 4.066 & 14.6 & 2.9 & No \\
17064 1999GX16 & 2.34 & 0.115 & 6.609 & 13.8 & 4.2 & Yes \\
28160 1998VC11 & 2.20 & 0.084 & 4.93 & 14.9 & 2.5 & No \\
28517 2000DD7 & 2.19 & 0.092 & 7.863 & 13.2 & 5.5 & No \\
31692 1999JQ31 & 2.42 & 0.072 & 5.918 & 13.8 & 4.2 & No \\
31953 2000GZ125 & 2.26 & 0.041 & 7.556 & 15.0 & 2.4 & No \\
32940 1995UW4 & 2.18 & 0.094 & 4.405 & 14.5 & 3.0 & No \\
61235 2000OT15 & 2.40 & 0.111 & 7.192 & 14.4 & 3.2 & No \\
64276 2001TW218 & 2.30 & 0.168 & 6.575 & 15.4 & 2.0 & No \\
\hline
\end{tabular}

Notes. The proper orbital parameters are taken from the astdys web site (http://hamilton.dm.unipi.it/astdys/) and the diameters are computed assuming an albedo of 0.3 as in Marchi et al. (2010). The family membership has been defined as in Nesvorný et al. (2008).

Table 2. Observational circumstances for the selected asteroids.

\begin{tabular}{lccccc}
\hline \hline Asteroid & Date & RA & Dec & Exp. time (s) & Visual Mag. \\
\hline 4383 Suruga & $2010-03-18$ & $14: 59: 13$ & $-07: 38: 47$ & 11206 & 17.4 \\
5498 Gustafsson & $2010-03-16$ & $09: 13: 12$ & $13: 14: 20$ & 2240 & 18.5 \\
5560 Amytis & $2010-03-21$ & $09: 40: 48$ & $23: 14: 57$ & 1120 & 16.4 \\
6563 Steinheim & $2010-03-18$ & $10: 55: 31$ & $08: 24: 14$ & 900 & 17.3 \\
6976 Kanatsu & $2010-03-18$ & $10: 05: 17$ & $23: 35: 36$ & 900 & 17.4 \\
8761 Crane & $2009-11-23$ & $00: 31: 05$ & $06: 40: 00$ & 1800 & 17.8 \\
9147 Kourakuen & $2010-03-19$ & $15: 04: 48$ & $-09: 12: 26$ & 1800 & 17.6 \\
9531 Jean Luc & $2010-03-22$ & $12: 19: 21$ & $-02: 51: 15$ & 1800 & 17.3 \\
10614 1997UH1 & $2010-03-20$ & $14: 10: 18$ & $-08: 38: 21$ & 1800 & 17.8 \\
11764 Benbaillaud & $2009-11-23$ & $02: 19: 55$ & $+19: 02: 52$ & 1800 & 17.1 \\
17064 1999GX16 & $2010-03-21$ & $16: 37: 20$ & $-12: 36: 33$ & 1120 & 17.7 \\
28160 1998VC11 & $2010-03-19$ & $13: 05: 13$ & $10: 01: 46$ & 1800 & 17.6 \\
28517 2000DD7 & $2010-03-17$ & $07: 11: 49$ & $32: 06: 10$ & 2240 & 17.5 \\
31692 1999JQ31 & $2010-03-19$ & $12: 15: 12$ & $08: 33: 34$ & 1800 & 16.9 \\
31953 2000GZ125 & $2010-03-21$ & $13: 09: 24$ & $00: 22: 07$ & 2240 & 17.7 \\
32940 1995UW4 & $2010-03-19$ & $13: 10: 22$ & $02: 03: 50$ & 1800 & 17.6 \\
61235 2000OT15 & $2010-03-17$ & $12: 21: 39$ & $04: 59: 57$ & 2240 & 18.1 \\
64276 2001TW218 & $2010-03-19$ & $10: 29: 56$ & $17: 01: 17$ & 1800 & 18.0 \\
\hline
\end{tabular}

Notes. The exposure time in seconds is derived by summing several acquisitions of each asteroid.

\section{Spectral analysis}

\subsection{NIR spectra}

The obtained near-infrared (NIR) spectra of the selected set of asteroids can be analyzed to derive their mineralogical surface properties. The obtained spectra are shown in Fig. 1.

The spectra of asteroids (8761) Crane and (11764) 1999GX16 stop at $2.25 \mu \mathrm{m}$ because of the different instrumental set-up used during their observations on November 2009 (Discretional Director Time - DDT). Some asteroids show spectra with a low $\mathrm{S} / \mathrm{N}$ in the region $1.8-1.9 \mu \mathrm{m}$. In these cases, the second pyroxene band can be affected by large errors. As one can see in Fig. 1, all spectra are characterized by the typical absorption bands near 1 and $2 \mu \mathrm{m}$ which are indicative of the presence of pyroxene.

\subsection{Taxonomy of the observed objects}

A number of criteria have been used to identify a V-type asteroid from its colors and spectral data (Bus \& Binzel 2002; DeMeo et al. 2009). According to DeMeo et al. (2009), V-type asteroids are characterized by a strong and narrow absorption band at $1 \mu \mathrm{m}$ (hereafter BI) and a second strong and wider band at $2 \mu \mathrm{m}$ (hereafter BII). The classes R, Q, and $\mathrm{O}$ are similar to the $\mathrm{V}$ one, but some differences can be identified. The R-class asteroids present a 1- $\mu \mathrm{m}$ feature broader than the V-type and a shape more similar to an S-type except for the deeper features. The spectra of the O-class asteroids have a very rounded and deep bowl-shaped absorption at $1 \mu \mathrm{m}$ and a strong absorption feature at $2 \mu \mathrm{m}$. The Q-class is characterized by a deep and distinct $1 \mu \mathrm{m}$ absorption feature, with evidence of another feature near $1.3 \mu \mathrm{m}$ and a $2 \mu \mathrm{m}$ feature with varying depths among objects. 

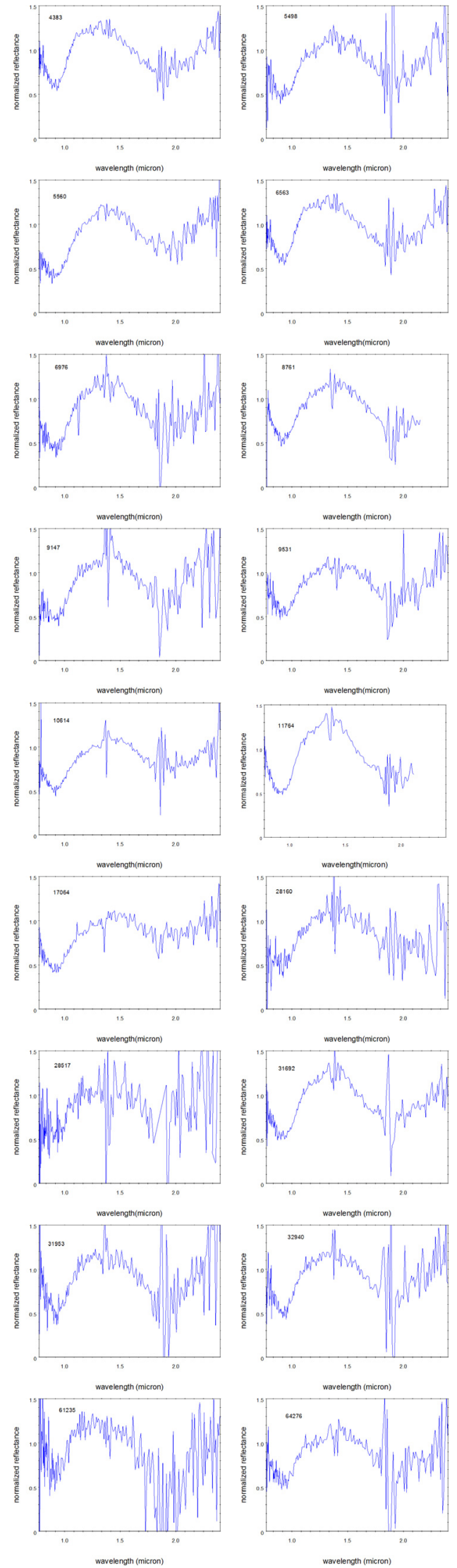

Fig. 1. Near-infrared spectra of the observed asteroids. All spectra are normalized to unity at $1.6 \mu \mathrm{m}$. The spectra of (8761) Crane and (11764) 1999GX16 stop at $2.25 \mu \mathrm{m}$ because of the different instrumental setup used during their observations on November 2009 (Discretional Director Time - DDT).

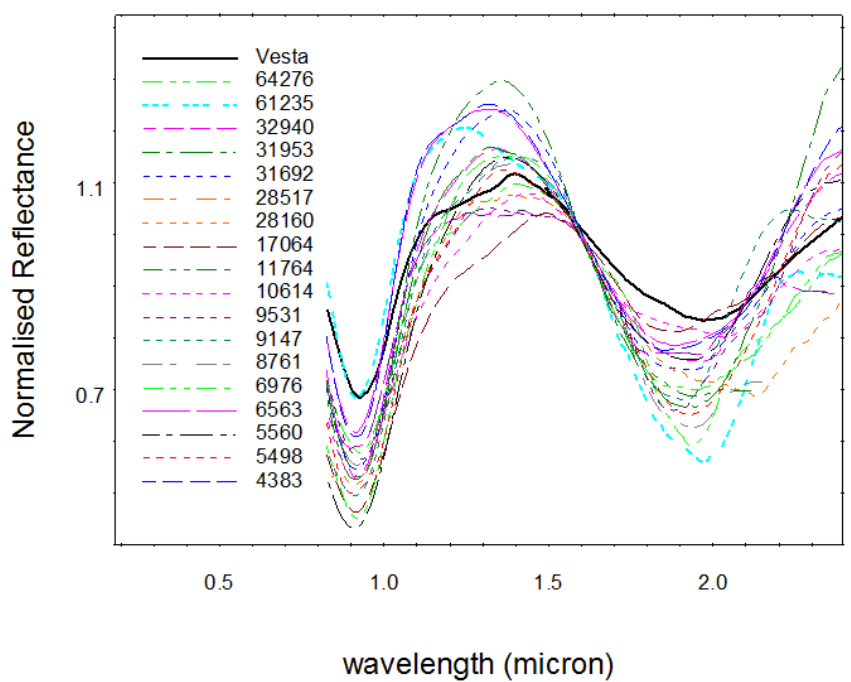

Fig. 2. Near-infrared smoothed spectra of the observed asteroids. All spectra are normalized to unity at $1.6 \mu \mathrm{m}$. For comparison, the (4) Vesta spectrum taken from the SMASS survey (Burbine \& Binzel 2002; Xu et al. 1995) is also plotted.

All spectra of the asteroids reported here show two prominent absorption features at 1 and $2 \mu \mathrm{m}$ that are typical of V-class asteroids. However, differences in the positions of the centers of the absorption bands, the band strengths, the band widths, and the slopes of the NIR continuum are observed, which indicates variations in the asteroid mineralogy.

Most of the spectra show absorption bands deeper than (4) Vesta, confirming that V-type asteroids have a stronger $1 \mu \mathrm{m}$ band (Florczak et al. 2002). From Fig. 2 it also appears that all observed asteroids have a stronger second pyroxenes band than (4) Vesta. Asteroid (17064) 1999GX16 shows a quite broad $1 \mu \mathrm{m}$ band which means that this asteroid is possibly an R-type object.

The same can be said for asteroid (10614) 1997UH1. However, both asteroids are classified as V-type by the Bus-DeMeo taxonomy (http://smass.mit.edu/ busdemeoclass.html). Asteroid (61235) 2000OT15 displays a quite unusual spectrum especially around 1.3-1.4 $\mu \mathrm{m}$, where a shoulder appears with a very prominent Band II, deeper than Band I. A similar spectral behavior at $1.3-1.4 \mu \mathrm{m}$ can be found in meteorites (Burbine et al. 2009).

The inferred mineralogy of the observed asteroids will be discussed in the following sections, but it is important to recall here that the observed asteroids have been selected as candidates to be V-types based only on their SDSS colors. Because indeed all seem to belong to the V-types, this is another confirmation that the methods developed to infer the distribution of basaltic asteroids are quite robust.

\subsection{Band parameters}

Band analysis techniques (Gaffey et al. 2002; Sunshine \& Pieters 1993) are useful to characterize spectral data and can be used to infer the associated mineralogy. The band minima, centers, depths, widths, and areas have been traditionally used for this purpose. The most useful are the minima and central wavelength of the 1 and $2 \mu \mathrm{m}$ olivine-pyroxene absorption features, defined as the wavelength position of the point of lowest reflectance before and after the removal of the continuum, respectively (Cloutis \& Gaffey 1991). The continuum, on the other hand, is defined by the linear fit between the maxima near 0.7 , 
Table 3. Spectral parameters.

\begin{tabular}{lcccc}
\hline \hline Asteroid & BI $\min (\mu \mathrm{m})$ & BI center $(\mu \mathrm{m})$ & BII $\min (=$ BII center $)(\mu \mathrm{m})$ & Band separation $(\mu \mathrm{m})$ \\
\hline 4383 & $0.913 \pm 0.005$ & $0.920 \pm 0.009$ & $1.91 \pm 0.01$ & $1.00 \pm 0.01$ \\
5498 & $0.920 \pm 0.002$ & $0.927 \pm 0.006$ & $1.92 \pm 0.01$ & $1.00 \pm 0.01$ \\
5560 & $0.905 \pm 0.005$ & $0.912 \pm 0.009$ & $1.90 \pm 0.01$ & $0.99 \pm 0.01$ \\
6563 & $0.924 \pm 0.006$ & $0.93 \pm 0.01$ & $1.91 \pm 0.01$ & $0.99 \pm 0.01$ \\
6976 & $0.911 \pm 0.003$ & $0.918 \pm 0.007$ & $1.93 \pm 0.01$ & $1.02 \pm 0.01$ \\
8761 & $0.925 \pm 0.005$ & $0.932 \pm 0.009$ & $1.95 \pm 0.01$ & $1.02 \pm 0.01$ \\
9147 & $0.908 \pm 0.001$ & $0.915 \pm 0.005$ & $1.87 \pm 0.01$ & $0.98 \pm 0.01$ \\
9531 & $0.930 \pm 0.001$ & $0.937 \pm 0.006$ & $1.94 \pm 0.01$ & $1.01 \pm 0.01$ \\
10614 & $0.919 \pm 0.003$ & $0.926 \pm 0.007$ & $1.95 \pm 0.01$ & $1.03 \pm 0.01$ \\
11764 & $0.920 \pm 0.005$ & $0.927 \pm 0.009$ & $1.97 \pm 0.01$ & $1.05 \pm 0.01$ \\
17064 & $0.919 \pm 0.005$ & $0.926 \pm 0.009$ & $1.94 \pm 0.01$ & $1.02 \pm 0.01$ \\
28160 & $0.90 \pm 0.01$ & $0.907 \pm 0.014$ & $2.02 \pm 0.03$ & $1.12 \pm 0.03$ \\
28517 & $0.908 \pm 0.005$ & $0.915 \pm 0.009$ & $1.90 \pm 0.02$ & $0.98 \pm 0.01$ \\
31692 & $0.920 \pm 0.003$ & $0.927 \pm 0.007$ & $1.93 \pm 0.01$ & $1.01 \pm 0.01$ \\
31953 & $0.929 \pm 0.005$ & $0.936 \pm 0.009$ & $1.92 \pm 0.01$ & $0.99 \pm 0.01$ \\
32940 & $0.926 \pm 0.001$ & $0.933 \pm 0.005$ & $1.91 \pm 0.01$ & $0.98 \pm 0.01$ \\
61235 & $0.932 \pm 0.002$ & $0.939 \pm 0.006$ & $1.93 \pm 0.02$ & $0.99 \pm 0.02$ \\
64276 & $0.925 \pm 0.002$ & $0.932 \pm 0.006$ & $1.96 \pm 0.01$ & $1.04 \pm 0.01$ \\
\hline
\end{tabular}

1.4 and $2.4 \mu \mathrm{m}$ and for its correct evaluation a spectrum is required that encompasses the two maxima, at $0.7 \mu \mathrm{m}$ and $1.4 \mu \mathrm{m}$. The asteroids reported here have been observed only in the NIR, typically from 0.75 to $2.5 \mu \mathrm{m}$, consequently a correct evaluation of the continuum between $0.7 \mu \mathrm{m}$ and $1.4 \mu \mathrm{m}$ cannot be achieved. For this reason, we will compute only band minima below and apply corrections based on laboratory data and previous observations to derive the band center, using simple relations between band minima and band centers found by Cloutis \& Gaffey (1991), as explained below. We recall that we used a similar approach in our previous paper (De Sanctis et al. 2011).

Using the full NIR spectra, we determined the position of the two minima near 0.9 and $2.0 \mu \mathrm{m}$. These values have been calculated by fitting a second order polynomial to the spectral curve in a small region of the minimum. We fitted the spectral region between 0.8 and $1.1 \mu \mathrm{m}$ for BI and between 1.6 and $2.3 \mu \mathrm{m}$ for BII. For asteroids (8761) Crane and (11764) Benbaillaud, the BII minimum was evaluated with a fit in the range 1.65 and $2.25 \mu \mathrm{m}$, because their spectra stop at $2.25 \mu \mathrm{m}$ owing to a different set up during their observations.

Owing to the sometimes apparently quite noisy spectra, we carefully checked the band minima and the associated errors using the spectra derived from different standard stars. We estimated the band minima using a method similar to that of Storm et al. (2007), repeating the fit by increasing each time the number of points in the band to fit and deriving the minima. Then, we averaged all corresponding wavelengths to obtain a value for the Band minima, and took the standard deviation of all corresponding wavelengths to obtain the value for the Band error bar.

We also verified the adopted method on data previously acquired at the TNG (Duffard et al. 2004), where both the visible and the near-infrared spectra for each asteroid were acquired during the same observing run. For these spectra, the difference between $\mathrm{BI}$ minimum and $\mathrm{BI}$ center is in the range $0.003-0.01 \mu \mathrm{m}$, with an average value of $0.007 \mu \mathrm{m}$. So, finally, we adopted the correction

$\mathrm{BI}$ center $=\mathrm{BI}$ minimum $+0.007 \pm 0.004 \mu \mathrm{m}$

to derive the $\mathrm{BI}$ center from the $\mathrm{BI}$ minimum.

Concerning the difference between BII minimum and BII center, we verified that the removal of the continuum does not have large effects on the band center position. This is because the slopes of the BII continuum are nearly flat and the BII center is within the error of the BII minimum. A similar approach has been adopted by other authors (Burbine et al. 2009; Moskovitz et al. 2010).

No temperature corrections to band centers were applied because they are very small, on the order of 0.0001-0.002 micron for DBI and about 0.01 micron for DBII (Burbine et al. 2009) and are within the errors given in our measurements. All computed parameters are provided in Table 3.

The band parameters are diagnostic of the mineralogy present on the surface of the observed asteroids. The relationship between these spectral parameters and the mineralogy, particularly pyroxene and olivine, has been studied in various papers over the last years (Adams 1974; King \& Ridley 1987; Cloutis \& Gaffey 1991; Klima et al. 2007; Gaffey et al. 2002). In the following section, we will discuss the different mineralogical properties of the observed asteroids as disclosed by the computed spectral parameters. Please note that we will not discuss the mineralogy associated to asteroid (28160) 1998VC11 and (28517) 2000DD7 because the low S/N of their spectra leads to large errors in the determination of the pyroxene bands and to unreliable values.

\subsection{Inferred mineralogy}

Band centers are among the most important diagnostic parameters of the mineralogy of the sample. It is well known that most pyroxenes and the basaltic achondrites show a strong correlation between the position of BI and BII centers and the associated mineralogy (Gaffey 1976; Adams 1974; Cloutis \& Gaffey 1991). Orthopyroxene bands shift to longer wavelengths with increasing amounts of iron, whereas clinopyroxene bands shift to longer wavelength with increasing calcium content (Klima et al. 2007).

The pyroxene composition, i.e., the molar $\mathrm{Ca}$ [Wo] and $\mathrm{Fe}[\mathrm{Fs}]$ contents, can be obtained from the values of the absorption band centers near 1 and $2 \mu \mathrm{m}$ using equations derived by exhaustive laboratory calibrations performed by several authors (Adams 1974; King \& Ridley 1987; Cloutis \& Gaffey 1991). These equations have been recompiled for pyroxenes (Gaffey et al. 2002) and for HED meteorites (Burbine et al. 2007) and are used here to derive the [Wo] and [Fs] contents for the observed asteroids. It is noteworthy that these equations are suitable for 


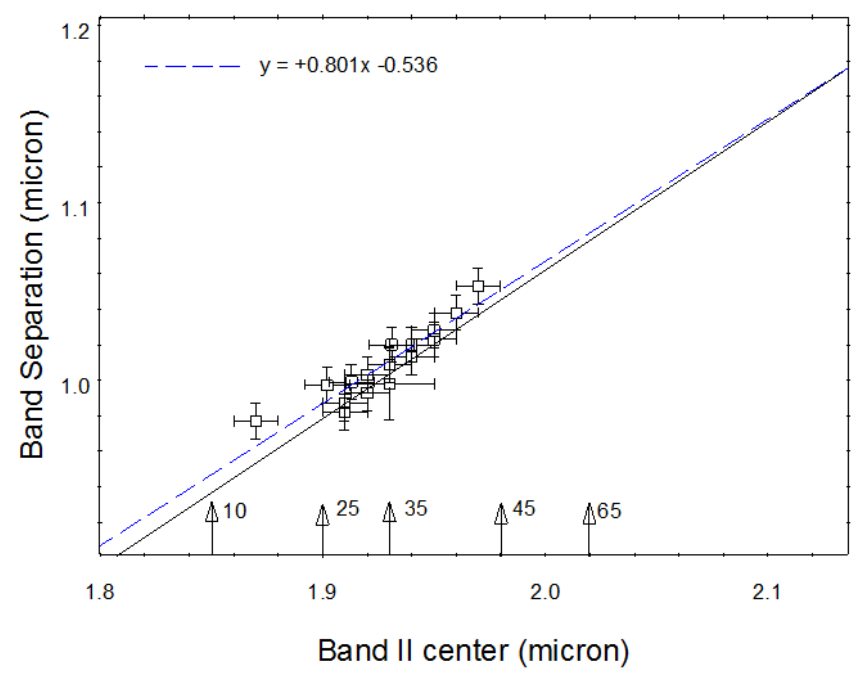

Fig. 3. Band separation vs. BII center. The arrows along the horizontal axis indicate the iron content (mole percent ferrosilite). The dashed line represents a linear least-squares fit to our data while the continuous line reproduces the value given by Cloutis et al. (1990).

spectra of pure pyroxene samples, of mixtures of olivine and orthopyroxene, and of HED meteorites (Gaffey et al. 2007). The Burbine et al. (2007) formulas only work on the very restricted pyroxene mineralogies found in HEDs, whereas the Gaffey et al. (2002) formulas are reliable on a much wider range of pyroxene mineralogies. Here we applied both formulas to obtain a better insight on the mineralogy of the observed asteroids. The errors on the molar content obtained using these formulas are on the order of $\pm 1-4$ according to Burbine et al. (2007) and on the order of $\pm 4-5$ according to Gaffey et al. (2002). Each formula provides a slightly different value, so we listed in Table 4 the range of values for [Wo] and [Fs] contents obtained for each asteroid. We fixed the associated errors as the highest possible values given by the adopted formulas. It is important to note, however, that if the band position uncertainty is large, the range for [Fs] and [Wo] contents and associated errors can be even larger.

Another way to infer the iron content is using the "band separation" plot. According to Cloutis et al. (1990), laboratory calibration indicates that a separation of the BI and BII minima (band separation) as a function of wavelength position of the BII minimum for orthopyroxenes shows a linear trend with both parameters increasing with increasing iron content.

Accordingly, we use plot to constrain the iron content in our sample. In Fig. 3 our data are presented along with a linear fit to it and, for comparison, the one from Cloutis et al. (1990). Evidently the two fits are very similar and indicate an iron content between $25 \mathrm{wt} \%$ and $45 \mathrm{wt} \%$, according to the laboratory calibrations. These values agree well with the computed values given in Table 4. It is important, however, to mention that these calibrations are true for a number of low aluminum orthopyroxenes and are not valid for mixtures of olivine, metal, and orthoand clino-pyroxenes.

\section{Discussion}

\subsection{Comparison of the spectral parameters of HEDs, V-type asteroids, and (4) Vesta}

The spectral parameters obtained in the previous section can be directly compared with those for HED as derived in De Sanctis et al. (2011). In Fig. 4 we plot the asteroid band centers and the

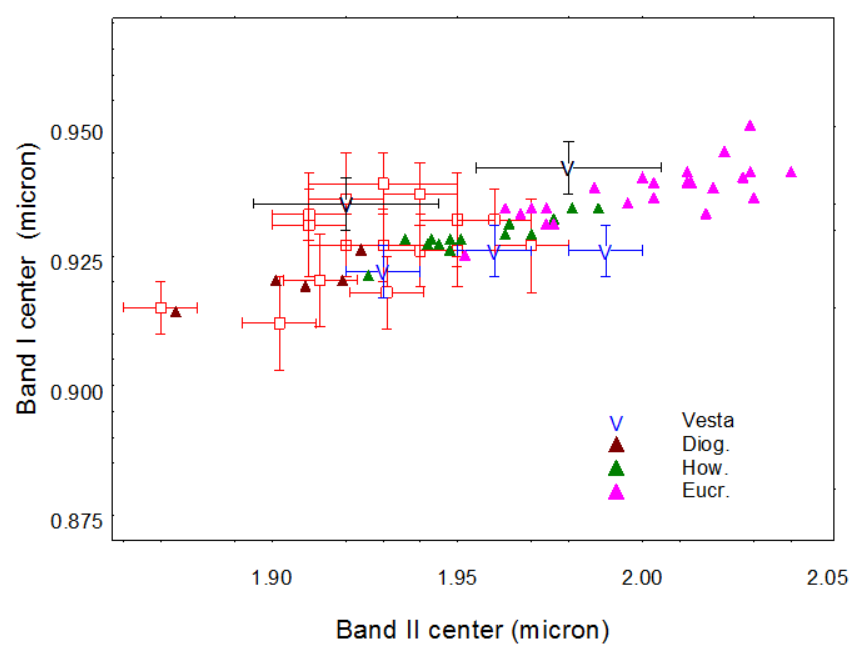

Fig. 4. Values of BI center vs. BII center of the observed sample of V-type asteroids (red squares) and HEDs (triangles). The HED values are from De Sanctis et al. (2011). The V symbols indicate (4) Vesta: black from Gaffey (1997) (the two points represent the maximum and the minimum values over a rotational periods with the associated errors), and blue from Vernazza et al. (2005).

Table 4. Molar contents for the observed asteorids.

\begin{tabular}{ccc}
\hline \hline Asteroid & $\begin{array}{c}{[\mathrm{Wo}]} \\
\pm 4\end{array}$ & $\begin{array}{c}{[\mathrm{Fs}]} \\
\pm 5\end{array}$ \\
\hline 4383 & $3-4$ & $24-29$ \\
5498 & $6-7$ & $32-34$ \\
5560 & $1-2$ & $24-26$ \\
6563 & $7-9$ & $29-35$ \\
6976 & $3-5$ & $30-34$ \\
8761 & $8-9$ & 39 \\
9147 & $0.5-2$ & $22-24$ \\
9531 & $9-12$ & $39-40$ \\
10614 & 7 & $36-39$ \\
11764 & $7-8$ & $38-45$ \\
17064 & $6-7$ & $35-36$ \\
31692 & $6-7$ & $34-35$ \\
31953 & $8-11$ & 38 \\
32940 & $7-10$ & $28-35$ \\
61235 & $9-12$ & $38-41$ \\
64276 & 9 & $41-43$ \\
\hline
\end{tabular}

HED band centers. The latter are derived from RELAB spectra of HED meteorite powders with grain sizes smaller than $25 \mu \mathrm{m}$ (De Sanctis et al. 2011).

Figure 5 shows the calculated [Fs] and [Wo] contents for the observed V-type asteroids vs. the ranges found for the HEDs. The average Wo and Fs contents are, respectively, $3.2 \pm 1.5$ and $26 \pm 5$ for diogenites, $7.2 \pm 3$ and $40 \pm 8$ for howardites and $11.5 \pm 5.7$ and $50 \pm 13$ for eucrites (De Sanctis et al. 2011). Many objects observed in the present work occupy the region with relatively low values of $\mathrm{BI}$ and $\mathrm{BII}$ centers, which is indicative of a low Ca content $(<10 \%$ Wo). Few of them are clearly in the region of diogenites. The diogenites are orthopyroxenites, and therefore contain Ca-poor (low Wo) pyroxenes. The region of eucrites is the one that overlaps less with the observed asteroids. We found a group of asteroids that are shifted toward Band I centers that are slightly higher than those of the HEDs (Fig. 4), the reason for which is not clear. However, BI vs. BII of these asteroids match one of the values reported for Vesta. 
M. C. De Sanctis et al.: Mineralogical characterization of V-type asteroids
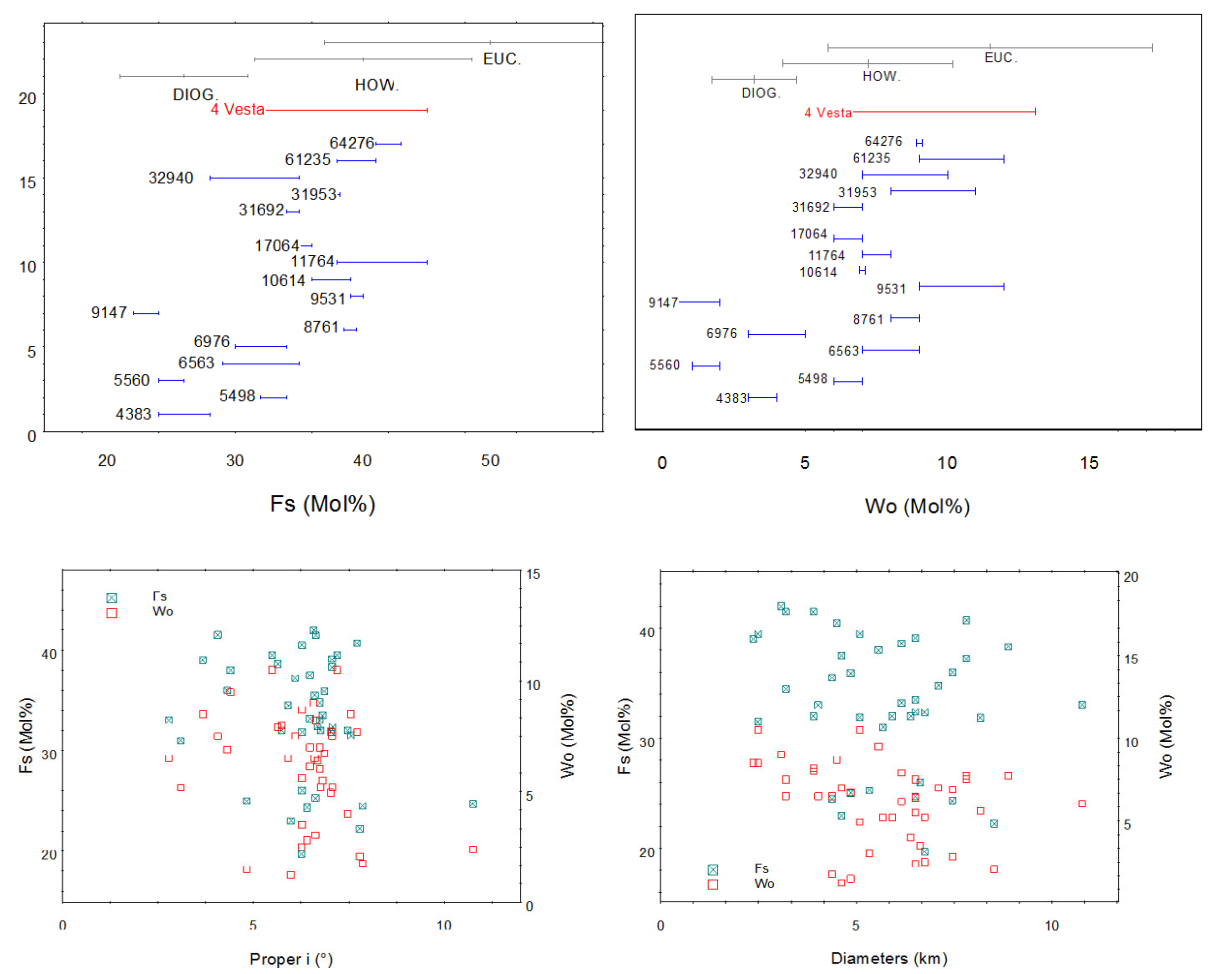

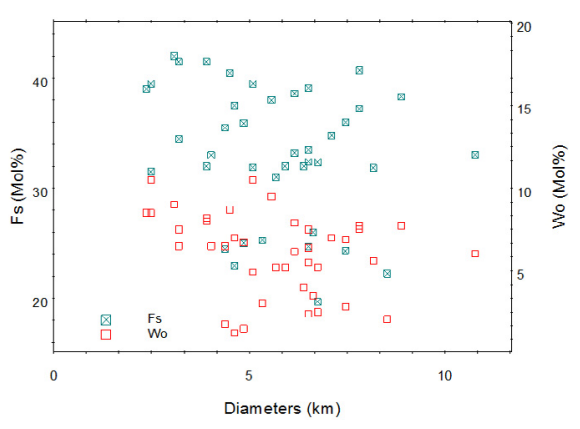

Fig. 5. Ranges for the eucrites, howardites, and diogenites meteorites derived in De Sanctis et al. (2011) compared with those for the observed asteroids. Left: ferrosilite [Fs]; right: wollastonite [Wo]. The errors, \pm 5 and \pm 4 , in the determination of $[\mathrm{Fs}]$ and [Wo] are not shown in the plots.

Fig. 6. Left panel: ferrosilite [Fs] and wollastonite [Wo] vs. proper inclination. Right panel: ferrosilite [Fs] and wollastonite [Wo] vs. diameters. In the diagrams the average values of the $[\mathrm{Fs}]$ and $[\mathrm{Wo}]$ ranges (Table 4) are plotted.
The BI vs. BII plot shows that (4) Vesta (values from Gaffey 1997; and Vernazza et al. 2005) lies in the region between diogenites and eucrites (mainly howardites region). The (4) Vesta data, however, indicate a quite large spectral variability. In the data from Gaffey (1997) the range of the BI and BII centers of (4) Vesta falls between the eucrite and diogenite regions, slightly above the HEDs trend. Vernazza et al. (2005), instead, reported that BI and BII fall in the transition region between low- and high-calcium pyroxenes, close to the diogenites. The V-type asteroids examined here show a higher variability of band parameters compared to the HEDs values than (4) Vesta. This indicates that (4) Vesta and V-type asteroids have mineralogies that are not completely represented by the HEDs.

The observed objects have average [Fs] and [Wo] contents consistent mainly with howardites and diogenites. Only few asteroids seem to have mineralogies consistent only with eucritic materials. In general the formulas used to derive the [Fs] and [Wo] contents work well for laboratory spectra with very high $\mathrm{S} / \mathrm{N}$, for which it is easy to determine the pyroxenes bands position with small errors. For asteroids quite faint with low $\mathrm{S} / \mathrm{N}$ it is difficult to determine the exact band position and the spectral analysis is often affected by large errors. On the other hand, there are some asteroids that show a narrower range of possible mineralogies.

\subsection{Band parameters, sizes, and orbital parameters}

The orbital distribution of basaltic asteroids in the inner main belt suggests that (4) Vesta is their principal progenitor. However, many basaltic asteroids appear to be scattered all around the main belt, showing a wide distribution of orbits, which may be a signature that not all of them are necessarily linked to (4) Vesta. In order to investigate if objects sharing similar orbital parameters also show some common characteristics, we studied the possible correlation between band parameters and orbital properties. The objects presented in this paper all lie in the inner part of the main belt, as can be seen in Table 1. We also investigated the possible correlation between mineralogy and asteroid dimension. To enlarge the data set and increase the statistical significance of the analysis, we included the data presented in our previous article (De Sanctis et al. 2011) for a total data set of $41 \mathrm{~V}$-type asteroids (Table 5). It is important to note that all these asteroids have been observed with the same telescope and the same instrumental set-up, and the method applied to derive band centers and associated mineralogy is the same for the whole dataset.

We searched for a correlation between low-inclination V-type asteroids and compositions. The low-inclination $\mathrm{V}$-asteroids could be a distinct population of asteroids. The Nesvorný et al. (2008) dynamical simulations did not produce a sufficient number of Vestoid fragments with low-inclination orbits to explain the observed distribution of V-type asteroids in the inner main belt. The authors concluded that the lowinclination V-type objects can be asteroids that do not come from Vesta or are fragments from the surface of Vesta that were ejected at an epoch earlier than the main family-forming collision. If these hypotheses are true, the V-type asteroids in that region of orbital element may be spectroscopically distinct from Vesta-family members. The low-inclination region in the Nesvorný et al. (2008) simulations was referred to as Cell 2 and defined as $2.32<a<2.48,0.05<e<0.2,2<$ $i<6$. Unfortunately, our dataset counts only two asteroids in Cell 2, which prevents any significant statistical analyses on that cell. However, looking at the distribution of the [Fs] and [Wo] content with respect to inclination (Fig. 6), we can note a lack of low-inclination objects with low [Fs] and [Wo] content. However, because of the small number of low inclination objects, it is difficult to state if this tendency is real.

The plot of composition vs. asteroids size (here we are assuming the same albedo for the whole data set, meaning that 
Table 5. V-Type asteroids orbital parameters and associated mineralogy.

\begin{tabular}{|c|c|c|c|c|}
\hline Asteroid & $\begin{array}{c}a(\mathrm{AU}) \\
\text { non vesta }\end{array}$ & $\begin{array}{c}e \\
\text { family }\end{array}$ & $i\left(^{\circ}\right)$ & Mineralogy* \\
\hline 809 & 2.28 & 0.145 & 6.736 & $\mathrm{H}$ \\
\hline 956 & 2.30 & 0.158 & 6.408 & D \\
\hline 2763 & 2.40 & 0.179 & 4.318 & H-E \\
\hline 2851 & 2.48 & 0.120 & 7.793 & D \\
\hline 2912 & 2.29 & 0.118 & 6.742 & H-E \\
\hline 4383 & 2.42 & 0.055 & 6.275 & D \\
\hline 4796 & 2.36 & 0.141 & 3.095 & $\mathrm{H}$ \\
\hline 5498 & 2.24 & 0.098 & 2.786 & $\mathrm{H}$ \\
\hline 5560 & 2.28 & 0.118 & 4.824 & D \\
\hline 6406 & 2.28 & 0.127 & 7.712 & H-E \\
\hline 6563 & 2.29 & 0.097 & 5.745 & $\mathrm{H}$ \\
\hline 6976 & 2.33 & 0.120 & 7.475 & D-H \\
\hline 7148 & 2.29 & 0.100 & 5.641 & H-E \\
\hline 8693 & 2.41 & 0.122 & 6.269 & D-H \\
\hline 8761 & 2.24 & 0.096 & 3.675 & $\mathrm{H}$ \\
\hline 9147 & 2.19 & 0.108 & 6.892 & D \\
\hline 9531 & 2.23 & 0.138 & 5.492 & H-E \\
\hline 11764 & 2.19 & 0.070 & 4.066 & H-E \\
\hline 21238 & 2.54 & 0.137 & 10.754 & D \\
\hline 31692 & 2.42 & 0.072 & 5.918 & H-E \\
\hline 31953 & 2.26 & 0.041 & 7.556 & H-E \\
\hline 32940 & 2.18 & 0.094 & 4.405 & H-E \\
\hline 61235 & 2.40 & 0.111 & 7.192 & H-E \\
\hline \multirow[t]{2}{*}{64276} & 2.30 & 0.168 & 6.575 & H-E \\
\hline & vesta & family & & \\
\hline 1929 & 2.36 & 0.114 & 7.065 & H-E \\
\hline 1933 & 2.35 & 0.094 & 6.811 & $\mathrm{H}$ \\
\hline 2011 & 2.39 & 0.114 & 7.065 & H-E \\
\hline 2468 & 2.33 & 0.116 & 6.275 & $\mathrm{H}$ \\
\hline 3268 & 2.35 & 0.100 & 7.025 & H-E \\
\hline 3498 & 2.36 & 0.099 & 6.863 & H-E \\
\hline 3782 & 2.42 & 0.108 & 6.102 & H-E \\
\hline 3944 & 2.37 & 0.109 & 6.754 & D-H \\
\hline 3968 & 2.32 & 0.091 & 6.679 & $\mathrm{H}$ \\
\hline 4147 & 2.36 & 0.102 & 6.483 & $\mathrm{H}$ \\
\hline 4815 & 2.36 & 0.099 & 7.233 & H-E \\
\hline 4993 & 2.37 & 0.091 & 6.31 & $\mathrm{H}$ \\
\hline 6331 & 2.36 & 0.097 & 7.077 & H-E \\
\hline 10320 & 2.29 & 0.090 & 6.632 & H-E \\
\hline 10349 & 2.38 & 0.095 & 6.627 & D \\
\hline 10614 & 2.35 & 0.089 & 6.483 & H-E \\
\hline 17064 & 2.34 & 0.115 & 6.609 & H-E \\
\hline
\end{tabular}

Notes. ${ }^{(*)}$ Associated mineralogy according to the estimated [Fs] and [Wo] content.

the derived diameters are directly linked with absolute magnitude $H$ ), show that smaller asteroids have a higher content of [Fs] and [Wo] compared to the rest of the data set (Fig. 6). However, as for the low inclination asteroids, it is difficult to say if this trend is real or is the result of a statistical fluke.

\subsection{Difference between Vesta family and non-family members}

We analyzed the differences between Vesta family and non-Vesta family asteroids: the data set comprises 17 family asteroids and 24 non-family ones, with a cut-off velocity of $55 \mathrm{~m} / \mathrm{s}$ (Nesvorný, priv. comm.). In Fig. 7 BI vs. BII centers of Vesta family and non-family asteroids are reported.

It is difficult to draw a clear conclusion concerning the mineralogical differences between Vesta family and non-family members, but some trends can be found. Both the family and the

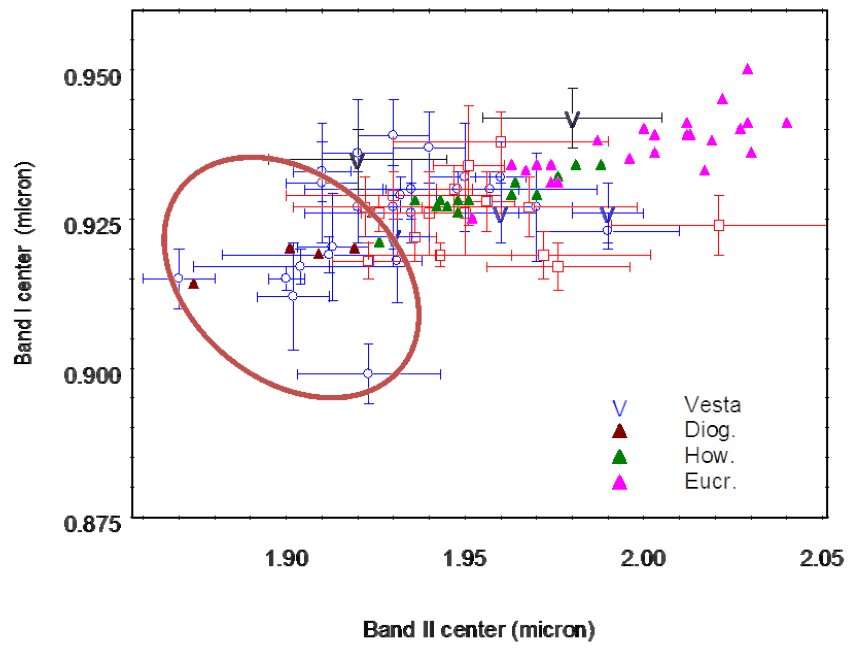

Fig. 7. Values of BI center vs BII center for our sample of asteroids and for HEDs. Blue dots represent asteroids that are not members of the Vesta dynamical family, red squares represent those that are. The V symbols indicate (4) Vesta: black from Gaffey (1997) (the two points represent the maximum and minimum values over a rotational periods with the associated errors), blue from Vernazza et al. (2005). The brown circle indicates the region where the diogenite-like asteroids are located.

non-family members have values consistent with the HEDs. However, the distribution of band centers for the two subsets are slightly different: the non-family objects show several elements in the lower part of the plots, corresponding to the diogenite region, while the family objects show a clear lack of elements in that region of the plot. Moreover, the non-family asteroids congregate in a small cluster in the region between $0.93-0.94 \mu \mathrm{m}$ and $1.91-1.94 \mu \mathrm{m}$, above the howardite region.

Apparently the non-family objects have band centers at slightly lower wavelengths (average BI $=0.924 \pm 0.02, \mathrm{BII}=$ $1.93 \pm 0.06$ ), suggesting a population richer in diogenitic material compared to the family members (average BI $=0.926 \pm$ 0.01 , BII $=1.95 \pm 0.05$ ).

This finding is supported by the inferred [Wo] and [Fs] content of the analyzed dataset. Nine non-family asteroids from a total of 24, have a [Wo] content consistent with diogenites, while the family members have a [Wo] content similar to howardites and eucrites, which confirms the results given in De Sanctis et al. (2011). Most of the observed asteroids, both family and nonfamily members, overlap very well with Vesta BI and BII values (Gaffey 1997; Vernazza et al. 2005), spanning wider ranges in BI and BII with respect to HEDs values. Only a small subgroup, the "diogenitic-like" asteroids, have band center values that are quite distinct from those of Vesta. Interestingly, as mentioned above, these asteroids are non-Vesta family members.

\subsection{Diogenitic asteroids}

We analyzed 41 V-type asteroids and found mineralogical differences among them, in particular, with band parameters similar to eucrites, howardites, and diogenites. Although previous works have reported an apparent lack of diogenitic material among the V-type asteroids in the inner main belt (Burbine et al. 2001; Moskovitz et al. 2008), this is not the case in our sample. This finding, already presented in our previous work (De Sanctis et al. 2011), has also been confirmed by Reddy et al. (2010), who discovered for the first time "a fragment of Vesta mantle" among the NEO population. 
Moscovitz et al. (2010) also present one object, (21238), that clearly is in the diogenite region, while several others (2851, 3155,26886 and 27343) are in-between the diogenites and howardites regions. According to these authors, it is quite plausible that the latter four asteroids are primarily composed of diogenitic material with some eucritic contamination on their surface. Two of these objects, (2851) and (21238), have also been observed by De Sanctis et al. (2011), who confirm the diogenitc nature of the asteroids' surface.

In Table 5 we report all analyzed asteroids with their family and meteorite association. Evidently, seven asteroids can be associated to diogenites, three to diogenite and/or howardite, ten to howardite, and the remaining to a mixture of howardite and eucrite. It is important to note that among the seven diogenitic-like asteroids, (956), (2851), (4383), (5560), (9147), (21238), and (10349), just the last one is classified as member of the Vesta dynamical family. These diogenitic-like asteroids have semi-major axes ranging from 2.19 $\mathrm{AU}$ up to $2.54 \mathrm{AU}$, i.e., from the inner to the intermediate main belt. Therefore, it seems that diogenitic material, even if relatively rare, is not concentrated in a unique region of the main belt and is present also among NEO population (Reddy et al. 2010). The inner belt diogenites could be linked to either Vesta or possibly other bodies in the inner belt, and the NEOs are most likely linked to Vesta as well (Reddy et al. 2010). Only one object (21238) is confidently not linked to Vesta. The distribution of diogenitic asteroids in the inner belt could be largely caused by the Yarkovsky drift and diogenitic asteoroids could be delivered to the NEO population through resonances.

\subsection{Comparison with previous works}

Ideally, the results presented here should be compared with those of previous works, but this is quite a complex task, as already noted by other authors (Duffard et al. 2006; Moscovitz et al. 2010). The greatest problem arises from subtle differences in the procedure adopted by the diverse authors to derive the band parameters, which makes reliable comparisons difficult. Beside this, we acknowledge that our results, although internally consistent, differ slightly from those of previous works. This deserves a deeper discussion.

First of all, werecall that although several papers deal wih V-types mineralogy, some are limited to the analysis of the first pyroxene band and visual slopes (e.g., Hiroi \& Pieters 1998; Burbine et al. 2001). Moreover, the studied objects are not uniform in terms of orbital parameters, spanning from NEO to middle belt asteroids. The only other paper that analyzes a dataset similar to ours is the one by Moskovitz et al. (2010), with a large number of V-type asteroids in the inner main belt and showing that the band parameters are consistent with those of the HED meteorites. Remarkably this is also the main result of the present paper.

One of the main results of Moskovitz et al. (2010) is related to the correlation found between band parameters and orbital parameters. According to their analysis, V-type asteroids in the inner main belt and low-inclination orbits $\left(i<6^{\circ}\right)$ tend to have their band center shifted to longer wavelengths. Unfortunately, our dataset lacks a significant number of objects with these orbital characteristics, which prevents us from confirming or contradicting this trend. We note, however, that in our sample there is a lack of low-inclination objects with low [Fs] and [Wo] content, which could indicate a trend similar to the one discussed by Moscovitz et al. (2010).
Another important result by Moskovitz et al. (2010) is the discovery of a group of asteroids with a Band I center slightly higher than those spanned by the HED. Again, this occurs as well in our sample, as shown in Fig. 6. As discussed above, the objects of this group have BI vs. BII consistent with values for (4) Vesta (Gaffey 1997). The reason for this displacement is unclear because higher $\mathrm{Ca}$ - and $\mathrm{Fe}$-abundances shift the band centers to longer wavelengths (Klima et al. 2007), and this should affect both Band I and Band II. According to Klima et al. (2007), the shift in just Band I could be caused by a cation larger than $\mathrm{Mg}$, such as $\mathrm{Ca}$, in the pyroxene.

The presence of possibly diogenitic asteroids in the Moscovitz et al. (2010) dataset has been discussed above.

\section{Conclusion}

We analyzed the spectra of $41 \mathrm{~V}$-type asteroids, 18 of which we gathered from new observations, inferring the mineralogy from the band parameters. Our measured band centers suggest a good correlation between those of the HEDs, (4) Vesta, and V-type asteroids.

Some asteroid band parameters are more consistent with those of HEDs, while some others are closer to Vesta's. We recall that the surface of (4) Vesta is not uniform, showing albedo variegation and spectral diversity (Binzel et al. 1997; Vernazza et al. 2005). This diversity has been interpreted as due to regions with differing mineralogies, like eucrite or diogenite-rich surface units (Gaffey 1997; Binzel et al. 1997). The V-type asteroids analyzed here show differences in their mineralogy with a prevalence of asteroids similar to diogenites and howardites, confirming the results found by De Sanctis et al. (2011). Some of them occupy a region with relatively low values of BI and BII centers, which is indicative of a low content of $\mathrm{Ca}(<10 \%$ Wo $)$ and consistent with diogenitic material. Previous authors have noted that there appears to be a lack of diogenetic V-type asteroids in the inner main belt (Burbine et al. 2001). However, we found asteroids with band parameters typical of diogenitic materials.

The identification of diogenite-like asteroids has important implications on their formation. Diogenites material originated from a plutonic layer deep in Vesta's lower crust/upper mantle (McSween et al. 2010) and it has been suggested that the lack of this mineralogy among the V-type asteroids could be due to the difficulty in excavating large fragments from depths (Moskovitz et al. 2010; Burbine et al. 2009). However, numerical simulations (Asphaug 1997) of the South Pole crater formation have shown that a large fraction of the excavated material would have come from Vesta's mantle and not its crust, because it has a diogenite-dominated composition. Thus, the identification of several diogenitic asteroids can help constraining the excavation mechanism.

Moreover, the discoveries of diogenite units (Gaffey 1997; Reddy et al. 2010; Li et al. 2010) on the surface of (4) Vesta support the possibility of large impact events capable of ejecting material from deep mantle layers.

It must be noted, however, that almost all "diogenite-like" asteroids sampled here are classified as non-Vesta family members. Dynamical simulations have shown that some asteroids outside the Vesta family could be former family members that migrated to their current positions via Yarkovsky effect and nonlinear secular and mean-motion resonances. In those cases, the non-family V-type asteroids sampled by our observations could, indeed, originate from Vesta. They are not recognized as part of the family because of a higher ejection velocity during the crater excavation and because of their subsequent dynamical evolution. 
Another option is that they are fragments from an older impact than the one that created the eucrite-like present Vesta family. In any case, we cannot exclude that some of these diogeniticlike non-family asteroids may represent distinct differentiated objects with no relation to Vesta.

From the band analysis we found that the family and the nonfamily members have values consistent with the HED distribution. However, the distribution of band centers for the two subsets are slightly different: the non-family objects show several values corresponding to the diogenite region, while the family objects show a clear lack in that region.

The search for correlations between spectroscopic characteristics and dynamical properties and diameters did not reveal unambiguously significant results even if we noted that the smaller asteroids have a composition richer in [Fs] and [Wo] than the larger asteroids. Fe-rich material is also found among the lowinclination V-type asteroids. However, owing to the relatively small data set, it is difficult to state the statistical relevance of these trends.

We observed and analyzed the spectra of $41 \mathrm{~V}$-type asteroids the main belt with the aim to find differences or trends among them. The obtained results indicate that

1. some asteroids present a diogenite-like mineralogy;

2. almost none of the diogenite-like asteroids are "classical" Vesta family members.

The above findings could have important implications for understanding the origin of basaltic material in the main belt. However, additional data should be obtained to confirm or refute the significance of these results. These data must be acquired with ground-based observations of other V-type asteroids, in particular those of non-family members. However, we expect that a tremendous step forward in the understanding the role of Vesta in the basaltic inventory in the main belt will be obtained by the Dawn spacecraft (Russell et al. 2007; Pieters et al. 2011) when it enters into orbit around Vesta in the summer of 2011, acquiring hyperspectral data from the visible to the infrared range (De Sanctis et al. 2010).

Acknowledgements. The authors thank Dr. A. Fiorenzano, Dr. F. Ghinassi and Dr. W. Boschin for their support during the observations in November 2009. The authors also thank Dr. E. Molinari, director of the TNG telescope, for awarding time to this project in the frame of the Director Discretional Time. D.L. and F.L.J. were supported by diverse fellowships and grants by CNPq, FAPERJ and CAPES. We also thank an anonymous referee for his/her helpful comments.

\section{References}

Adams, J. B. 1974, J. Geophys. Res., 79, 4829

Alvarez-Candal, A., Duffard, R., Lazzaro, D., \& Michtchenko, T. 2006, A\&A, 459,969

Arai, T., Tomiyama, T., Saiki, K., \& Takeda, H. 2008, LPSC XXXIX, 2465

Asphaug, E. 1997, Meteorit. Planet. Sci., 32, 965
Binzel, R. P., Gaffey, M. J., Thomas, P. C., et al. 1997, Icarus, 128, 95

Binzel, R. P., Masi, G., \& Foglia, S. 2006, DPS, 38, 71.06

Burbine, T. H., Buchanan, P. C., Binzel, R. P., et al. 2001, Meteorit. Planet. Sci., 36,761

Burbine, T. H., Buchanan, P. C., \& Binzel, R. P. 2007, 38th LPSC, 1338, 2117

Burbine, T. H., Buchanan, P. C., Dolkar, T., \& Binzel, P. 2009, Meteorit. Planet. Sci., 44, 1331

Bus, S. J., \& Binzel, R. P. 2002, Icarus, 158, 146

Carruba, V., Michtchenko, T. A., Roig, F., Ferraz-Mello, S., \& Nesvorný, D. 2005, A\&A, 441, 819

Carvano, J. M., Hasselmann, P. H., Lazzaro, D., \& Mothé-Diniz, T. 2010, A\&A, 510, A43

Cloutis, E. A., \& Gaffey, M. J. 1991, J. Geophys. Res., 96, 22809

Cloutis, E. A., Gaffey, M. J., Smith, D. G. W., \& Lambert, R. J. 1990, J. Geophys. Res., 95, 8323

DeMeo, F., Binzel, R. P., Slivan, S. M., \& Bus, S. J. 2009, PDSS, 114

De Sanctis, M. C., Coradini, A., Ammannito, E., et al. 2010, Space Sci. Rev. DOI:10.1007/s11214-010-9668-5

De Sanctis, M. C., Ammannito, E., Migliorini, A., et al. 2011, MNRAS, 412, 2318

Duffard, R., \& Roig, F. 2009, P\&SS, 57, 229

Duffard, R., Lazzaro, D., Licandro, J., et al. 2004, Icarus, 171, 120

Florczak, M., Lazzaro, D., \& Duffard, R. 2002, Icarus, 159, 178

Gaffey, M. J. 1976, J. Geophys. Res., 81, 905

Gaffey, M. J. 1997, Icarus, 127, 130

Gaffey, M. J., Cloutis, E. A., Kelley, M. S., \& Reed, K. L. 2002, in Asteroid III, ed. W. F. Bottke Jr., A. Cellino, P. Paolicchi, \& R. P. Binzel (Tucson: Univ. of Arizona Press), 183

Hammergren, M., Gyuk, G., \& Puckett, A. W. 2006, unpublished [arXiv: astro-ph/0609420]

Hardersen, P. S., Gaffey, M. J., \& Abell, P. A. 2004, Mineralogy of Asteroid 1459 Magnya and implications for its origin, Icarus, 167, 170

King, T. V. V., \& Ridley, I. W. 1987, J. Geophys. Res., 92, 11457

Klima, R. L., Pieters, C. M., \& Dyar, M. D. 2007, Meteorit. Planet. Sci., 42, 235

Ivezic, Z., Tabachnik, S., Rafikov, R., et al. 2001, AJ, 122, 2749

Larson, H. P., \& Fink, U. 1975, Icarus, 26, 420

Lazzarin, M., Marchi, S., Barucci, M. A., Di Martino, M., \& Barbieri, C. 2004, Icarus, 169, 373

Lazzaro, D., Michtchenko, T., Carvano, J. M., et al. 2000, Science, 288, 2033

Li, J.-Y., McFadden, L., Thomas, P., et al. 2010, Icarus, 208, 238

Licandro, J., Ghinassi, F., \& Testi, L. 2002, A\&A, 388, L9

Marchi, S., De Sanctis, M. C., Lazzarin, M., \& Magrin, S. 2010, ApJ, 721, L172

McCord, T. B., Adams, J. B., \& Johnson, T. V. 1970, Science, 168, 1445

McFadden, L. A., McCord, T. B., \& Pieters, C. 1977, Icarus, 31, 439

McSween, H. Y., Mittlefehldt, D. W., Beck, A. W., Mayne, R. G., \& McCoy, T. J. 2010, Space Sci. Rev., DOI:10.1007/s11214-010-9637-z

Mittlefehldt, D. W., McCoy, T. J., Goodrich, C. A., \& Kracher, A. 1998, in Planetary materials, ed. J. J. Papike, Mineral. Soc. Am., 4

Moskovitz, N. A., Jedicke, R., Gaidos, E., et al. 2008, Icarus, 198, 77

Moskovitz, N., Willman, M., Burbine, T., Binzel, R., \& Bus, S. 2010, Icarus, 208,773

Nesvorný, D., Roig, F., Gladman, B., et al. 2008, Icarus, 193, 85

Pieters, C. M., McFadden, L. A., Prettyman, T., et al. 2011, SSR, 2011, in press

Reddy, V., Gaffey, M. J., Kelley, M. S., et al. 2010, Icarus, 210, 693

Roig, F., \& Gil-Hutton, R. 2006, Icarus, 183, 411

Roig, F., Nesvorný, D., Gil-Hutton, R., \& Lazzaro, D. 2008, Icarus, 194, 125

Russell, C. T., Barucci, M. A., Binzel, R. P., et al. 2007, Adv. Sp. Res., 40, 193

Storm, S., Bus, S. J., \& Binzel, R. P. 2007, BAAS, 38, 448

Sunshine, J. M., \& Pieters, C. M. 1993, JGR, 98, 9075

Thomas, P. C., Binzel, R. P., Gaffey, M. J., et al. 1997, Science, 277, 1492

Vernazza, P., Mothé-Diniz, T., Barucci, M. A., et al. 2005, A\&A, 436, 1113

Vilas, F., Cochran, A. L., \& Jarvis, K. S. 2000, Icarus, 147, 119

Xu, S., Binzel, R. P., Burbine, T. H., \& Bus, S. J. 1995, Icarus, 115, 1 